\title{
Vapour-liquid coexistence for purely repulsive point-Yukawa fluids
}

\author{
Marjolein Dijkstra†§ and René van Roijł§ \\ † Centre Européen de Calcul Atomique et Moléculaire, Ecole Normale Supérieure de Lyon, 46, \\ Allée d'Italie, 69364 Lyon Cédex 07, France \\ $\ddagger$ Laboratoire de Physique (URA 1325 du CNRS), Ecole Normale Supérieure de Lyon, 46, Allée \\ d'Italie, 69364 Lyon Cédex 07, France
}

Received 21 October 1997, in final form 12 December 1997

\begin{abstract}
Using a simple mean-field theory and MC simulations we demonstrate the existence of a vapour-liquid transition in a fluid with purely repulsive Yukawa pair interactions and a density-dependent screening parameter. This transition does not fit into the classical Van der Waals picture, as the mechanism of liquid condensation is not caused by long-ranged attractions. The phase separation is, instead, driven by the enhanced screening at higher densities, which leads to ideal-gas behaviour at sufficiently high densities. A fluid at intermediate densities can thus reduce its free energy by separating into a dilute vapour and a high-density liquid.
\end{abstract}

\section{Introduction}

The occurrence of a vapour-liquid transition in the phase diagram of simple fluids is well known to be strongly related to the presence of long-ranged attractive interactions between the constituent particles. This relation was first put forward by Van der Waals in the late 19th century [1]. The basic ingredients of his mean-field theory are the assumptions of longranged attractions and short-ranged (excluded-volume) repulsions. With these assumptions, Van der Waals could explain many features of the liquid-vapour transition. Most notably, the theory reproduced - in accordance with experiments-a critical temperature, above which no liquid-vapour transition takes place. Below this critical temperature, however, a phase separation of the homogeneous fluid phase into a coexisting vapour and liquid is possible in an appropriate density regime. The theory predicts a vanishing critical temperature if there are no attractions between the particles. This implies that any non-zero temperature is above the critical temperature in a purely repulsive system, so no vapour-liquid coexistence appears in the phase diagram. In fact, it was only recently that the relationship between attractive interactions and the existence of liquid-vapour coexistence was substantially refined. This refinement, based on computer simulations [2] and density functional calculations [3], quantified the minimum range of the attractions required for a stable vapour-liquid transition as being about one sixth of the range of the repulsions. Shorter-ranged attractions are not sufficient. Despite such refinements, the relatively crude Van der Waals prediction, 'no attraction, no vapour-liquid coexistence', is still generally accepted. In this article, however, we challenge this statement, and show that a liquid-vapour transition is possible in the absence of any attractions.

$\S$ Present address: H H Wills Physics Laboratory, University of Bristol, Royal Fort, Tyndall Avenue, Bristol BS8 1TQ, UK. 
We wish to stress that the present article is one concerned with principle, since the model that we use is probably too idealized to allow for direct comparison with experiments. Nevertheless, the model is an extreme case of well-established models for colloidal suspensions, and does show a new mechanism for a vapour-liquid transition in a fluid with only repulsive interactions.

\section{The model}

The system that we consider is a limiting case of the well-established DLVO model for aqueous suspensions of charged colloidal particles [4]. According to the DLVO theory, the effective pair interaction between charged colloids is of the screened Coulomb form [4]. For two spherical colloidal particles of diameter $D$ and total charge $-Z e$ (with $e$ the proton charge), this pair potential $w(r)$ reads

$$
w(r)=\frac{Z^{2} e^{2}}{\epsilon}\left(\frac{\exp [\kappa D / 2]}{1+\kappa D / 2}\right)^{2} \frac{\exp [-\kappa r]}{r}
$$

with $\epsilon$ the dielectric constant of the suspending medium and $r$ the centre-to-centre separation between the two colloidal particles. The range of the potential is determined by the screening parameter $\kappa$, which is given in terms of the temperature $T$ and the total ionic strength $I$ by

$$
\kappa^{2}=\frac{4 \pi e^{2} I}{\epsilon k_{B} T}
$$

with $k_{B} T$ the thermal energy unit. If all of the micro-ions in the suspension are monovalent, $I$ equals the total number density of micro-ions, i.e. $I=Z \rho+2 \rho_{s}$, where $\rho$ is the colloid density, $Z \rho$ the counter-ion density and $\rho_{s}$ the density of pairs of added salt ions.

For $D=0$ and in the high-salt limit $2 \rho_{s} \gg Z \rho$ (where $\kappa$ is approximately fixed for all densities $\rho$ ) this model reduces to a point-Yukawa fluid with fixed screening length. This model was studied extensively by Robbins, Kremer and Grest [5]. An important result of reference [5] is that there are three phases in the phase diagram, namely fluid and facecentred cubic (FCC) and body-centred cubic (BCC) solid. For later reference we mention here that the density dependence of the melting temperature, i.e. the phase boundary between the fluid and either of the two crystalline phases, was shown to be accurately described by a linear fit to $\kappa \rho^{-1 / 3}$. We also note that it is not a surprise that no vapour-liquid transition was found in reference [5], since the pair interaction (1) is purely repulsive.

In this paper we study, also for $D=0$, the opposite low-salt limit $2 \rho_{s} \ll Z \rho$, so the pair interaction considered in the following reads

$$
v(r)=\frac{Z^{2} e^{2}}{\epsilon} \frac{\exp [-\kappa r]}{r}
$$

with

$$
\kappa^{2}=\frac{4 \pi e^{2} Z \rho}{\epsilon k_{B} T} .
$$

The point-Yukawa potential (3) is again purely repulsive, so one does not expect a liquidvapour transition in this simplified model of a salt-free colloidal suspension. In the following we show, however, that a fluid described by (3) and (4) behaves completely differently to the analogous one in the high-salt limit. In particular, we show that there is a vapour-liquid coexistence, while there is no crystalline phase at all for many values of $Z$. Clearly, the mechanism of the vapour-liquid coexistence differs from the standard one as described by Van der Waals, since there is no cohesive energy (normally provided by the long-ranged 
attractions) that drives the liquid condensation. Instead, the liquid condensation is driven by a new mechanism, which we discuss qualitatively below. We also show why one should not expect crystalline phases in the phase diagram.

\section{Theory}

The key difference between the high- and low-salt limits is the dependence of the pair potential (3) on the thermodynamic state defined by $\rho$ and $T$, through $\kappa$ (equation (4)). Here we argue qualitatively that this dependence is responsible for the liquid-vapour coexistence and the absence of solid phases. In order to analyse this dependence in detail, it is convenient to introduce the so-called Bjerrum length $\lambda=e^{2} / \epsilon k_{B} T$, the typical particle separation $a=\rho^{-1 / 3}$ and the dimensionless density $\rho^{*}=\rho \lambda^{3}$. We thus use $\lambda$ as an (irrelevant) unit of length and no longer treat $T$ as an independent variable that determines the strength of the interactions; instead, this role will be played by $Z$.

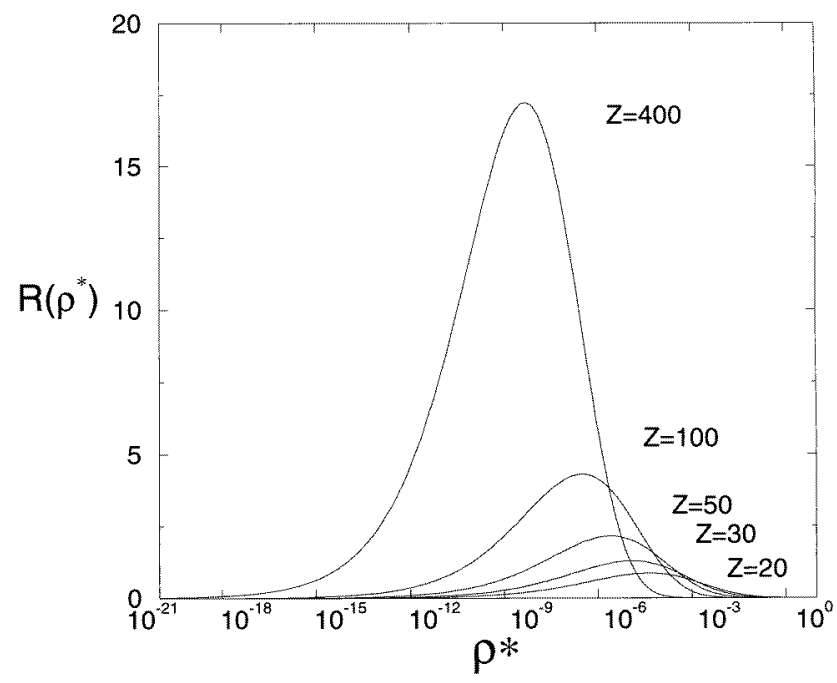

Figure 1. A measure of the 'importance' of the repulsions $R\left(\rho^{*}\right) \equiv v(a) / k_{B} T$ versus $\rho^{*}$ for purely repulsive point-Yukawa particles with $Z=20,30,50,100$ and 400.

We first define a simple dimensionless measure of the 'importance' of the repulsions by $R \equiv v(a) / k_{B} T$, which is the value of the pair interaction (in units of $k_{B} T$ ) at the typical separation $a$. Using (3) and (4) and $\kappa a=\sqrt{4 \pi Z}\left(\rho^{*}\right)^{1 / 6}$, it is easily checked that the density dependence of $R$ is given by

$$
R\left(\rho^{*}\right)=Z^{2}\left(\rho^{*}\right)^{1 / 3} \exp \left[-\sqrt{4 \pi Z}\left(\rho^{*}\right)^{1 / 6}\right] .
$$

In figure 1, we plot $R\left(\rho^{*}\right)$ for several values of $Z$. For all values of $Z$ we can distinguish clearly a maximum of $R$ as a function of $\rho^{*}$. This maximum becomes larger upon increasing $Z$. In the low- $\rho^{*}$ regime the algebraic prefactor $\left(\rho^{*}\right)^{1 / 3}$ is dominant, leading to an increasing importance of the repulsions as the density is increased. Such behaviour is to be expected for repulsive interactions. At high $\rho^{*}$, however, $R$ decreases with increasing $\rho^{*}$ due to the dominant exponential factor in (5). At such high densities the screening becomes exponentially stronger as the density increases, leading to a reduction of the repulsions. In view of this it is easy to imagine a coexistence between a dilute and a dense phase: 
the dilute phase exhibits only weak repulsions because of the large distances between the particles, while the dense phase exhibits weak repulsions due to the strong screening. At intermediate densities there is phase coexistence if the entropy cost of phase separation is more than compensated by the reduction of the repulsion.

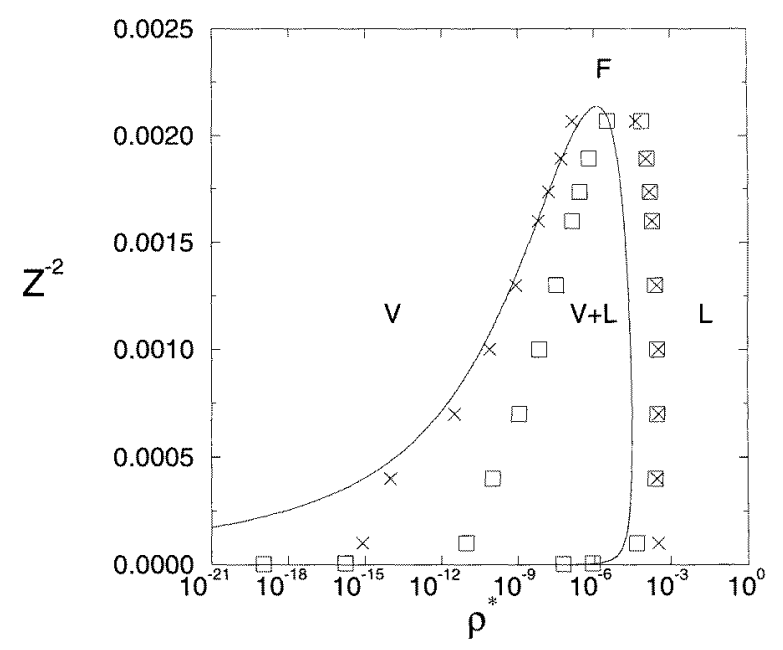

Figure 2. The phase diagram in the $\rho^{*}-Z^{-2}$ plane for a fluid of repulsive point-Yukawa particles, revealing vapour $(\mathrm{V})$, liquid $(\mathrm{L})$ and fluid $(\mathrm{F})$ phases and a vapour-liquid $(\mathrm{V}+\mathrm{L})$ coexistence region closed by a critical point. The mean-field prediction of the liquid-vapour phase boundary, based on (6) with $r_{0}=a$ (see the text), is denoted by —. The uncorrected simulated phase boundaries are denoted by $\square$ and the long-range-corrected ones by $\times$ (see the text).

The simple analysis above is essentially correct at high densities, where the pair potential decays exponentially over the range of the typical particle separation $a$. At low densities, however, the range of the potential can be much longer than $a$, so $v(a)$ is not necessarily a qualitative measure of the importance of the repulsions. It is then more appropriate to consider (a mean-field estimate of) the total potential energy $U_{p}$ in the system. Assuming that the pair correlation function vanishes at separations $r<r_{0}$ and equals unity for $r>r_{0}$, we can write

$$
\frac{U_{p}}{N k_{B} T}=\frac{\rho}{2 k_{B} T} \int_{r>r_{0}} \mathrm{~d} \boldsymbol{r} v(r)=\frac{Z}{2}\left(\kappa r_{0}+1\right) \exp \left(-\kappa r_{0}\right) .
$$

It is reasonable to choose $r_{0} \approx a$, so $\kappa r_{0} \approx \sqrt{4 \pi Z}\left(\rho^{*}\right)^{1 / 6}$. Then it follows directly that $U_{p} / N k_{B} T$ varies between $Z / 2$ in the low-density limit and 0 in the high-density limit. This result thus differs from the behaviour of $R\left(\rho^{*}\right)$, which vanishes not only at high densities but also at low densities. Nevertheless, also from the density dependence of $U_{p}$, one can understand a tendency for phase separation to occur: a fluid at intermediate densities can reduce its total free energy by phase separating into an almost ideal high-density liquid and an entropy-stabilized dilute vapour (with only a constant potential energy $Z k_{B} T / 2$ per particle, to be balanced by the high entropy of the sufficiently diluted vapour). This can be quantified numerically by considering the mean-field Helmholtz free energy $F$, consisting of the sum of the ideal-gas contribution $F_{i d}=N k_{B} T \log \rho^{*}$ and the potential energy $U_{p}$ in equation (6), i.e. $F=F_{i d}+U_{p}$. For the particular choice $r_{0}=a$ we solved the coexistence conditions (equal chemical potentials and pressures in the two phases) for a range of values of $Z$. The resulting vapour-liquid coexistence is shown as the full curve in the 
$\rho^{*}-Z^{-2}$ phase diagram of figure 2. This representation is chosen because $Z^{-2}$ plays the role of an effective temperature. The phase diagram thus resembles that of an ordinary fluid in the density-temperature plane, i.e. with a critical point below which liquid-vapour separation takes place, while above it the homogeneous fluid phase is stable for all densities. The critical point that follows from this simple mean-field analysis is characterized by $Z=21.6421$ and $\rho^{*}=1.3102 \times 10^{-6}$ with a mean-field critical exponent. As our purpose here is to give a simple explanation for the mechanism of the liquid-vapour separation, rather than to give a quantitative account, we are satisfied with the mean-field estimate of equation (6) and the resulting mean-field exponents. Of course, one could try to improve the mean-field description by using integral equations or density functional theory, but this is beyond the scope of this paper.

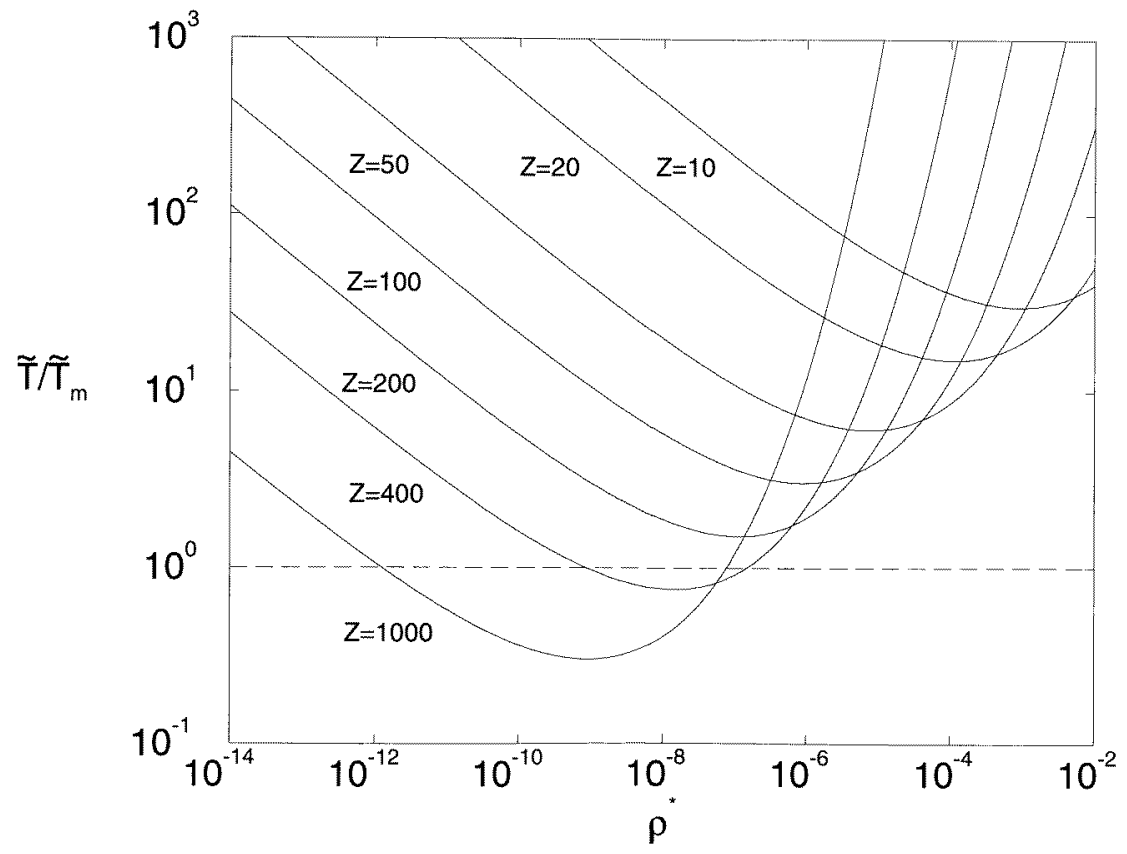

Figure 3. The ratio of the temperature $\tilde{T}$ and the fitted melting temperature $\tilde{T}_{m}$, discussed briefly in and below equation (7), as a function of the density $\rho^{*}$ for several values of $Z$. For $Z<300$ there is no regime with $\tilde{T} / \tilde{T}_{m}<1$ and hence no crystalline phase. For $Z>300$ there is a density regime with $\tilde{T} / \tilde{T}_{m}<1$, but these crystalline phases are unstable with respect to liquid-vapour coexistence.

We proceed with the theoretical analysis by showing that crystalline phases are not likely to occur for many, if any, values of $Z$. In order to see this one should realize that every thermodynamic state point, characterized by $\rho^{*}$ and $Z$ here, can be mapped onto the state variables $\kappa \rho^{-1 / 3}$ and $\tilde{T}$ used by Robbins et al in reference [5]. In this reference, the dimensionless temperature $\tilde{T}$ is defined as the ratio of the thermal energy unit $k_{B} T$ and a typical phonon energy of a FCC lattice $\{\boldsymbol{R}\}$ at density $\rho$. To be more specific, we have

$$
\tilde{T}=\frac{k_{B} T}{m \omega_{E}^{2} a^{2}}
$$

where the particle mass $m$ and the Einstein frequency $\omega_{E}$ satisfy $m \omega_{E}^{2}=\left(\kappa^{2} / 3\right) \sum_{\boldsymbol{R} \neq \mathbf{0}} v(\boldsymbol{R})$. 
Using equation (7), it is thus possible to check whether a value for $\tilde{T}$ that corresponds to a particular state point $\left(\rho^{*}, Z\right)$ is below or above the (dimensionless) melting temperature $\tilde{T}_{m}$, for which an explicit fit as a function of $\kappa \rho^{-1 / 3}$ is given in reference [5]. We have not used the more recent fit given in reference [6], which is based on computer simulations [7]. This fit, although supposedly more accurate, only holds in the interval $3<\kappa \rho^{-1 / 3}<7$, which is too small for our purposes. In figure 3 we plot the ratio $\tilde{T} / \tilde{T}_{m}$ as a function of $\rho^{*}$ for several values of $Z$. Clearly, for $Z<300$ we find $\tilde{T} / \tilde{T}_{m}>1$ for all $\rho^{*}$, so the system is always above the melting temperature and will not freeze into a FCC or BCC crystal. For $Z>300$ there are state points with $\tilde{T} / \tilde{T}_{m}<1$, which are thus mapped onto the FCC and BCC regions in the phase diagram of reference [5]. However, it turns out that for the values of $Z$ that we considered numerically, the relation $\tilde{T}<\tilde{T}_{m}$ only holds in a finite-density regime, embedded in the vapour-liquid coexistence region. It is therefore likely that the resulting crystalline phases are metastable with respect to the liquid-vapour phase separation.

In order to verify these theoretical considerations and to quantify the crude estimates, we present the results of MC calculations below.

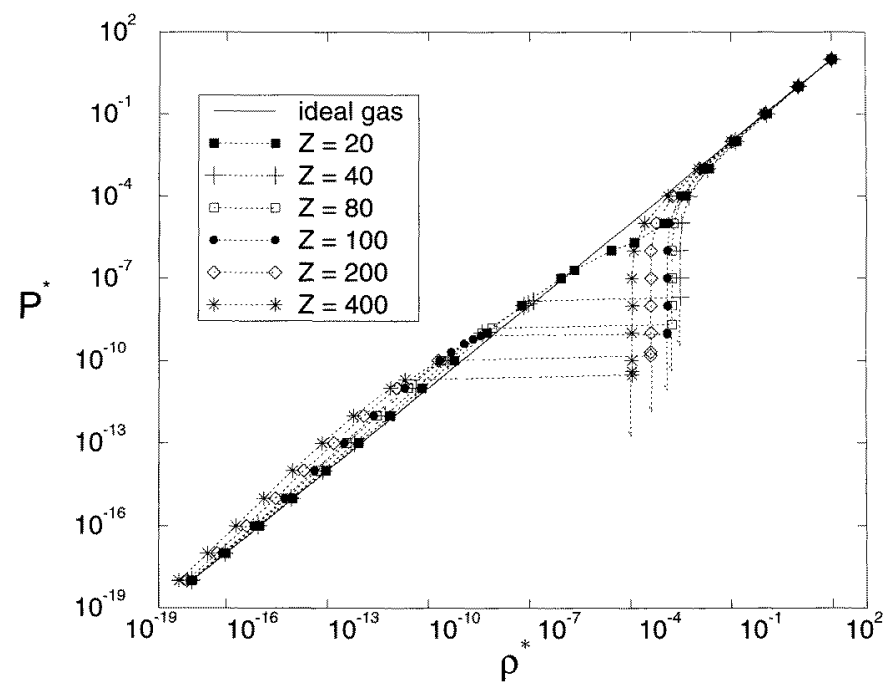

Figure 4. The equation of state for purely repulsive point-Yukawa fluids with $Z=$ 20, 40,80,100, 200 and 400 obtained from constant-pressure Monte Carlo simulations. The equation of state of an ideal gas is denoted by - For $Z \geqslant 40$, a plateau is found, indicating a first-order phase transition; for $Z=20$ there is no indication of a transition. The arrows indicate the onset of hysteresis.

\section{Simulations}

In order to find the liquid-vapour coexistence in the model under consideration we performed computer simulations. For several values of $Z$ we first performed constant-pressure Monte Carlo simulations, in which the volume $V$ of the system fluctuates [8]. We considered a system of $N=250$ particles at reduced pressures $P^{*}=\beta P \lambda^{3}$ ranging from $10^{-19}$ to $10^{2}$. Most runs consisted of 1000 cycles per particle per thermodynamic state point. In each cycle, we attempt a displacement of a random particle and a volume change of the 
system. For each pressure, we compute the averaged reduced density $\rho^{*}$. In figure 4 we plot the resulting $P^{*}$ as a function of $\rho^{*}$. When we gradually increase the pressure for $Z=40-400$, we find that the density increases smoothly initially, until it jumps to a much higher value. In contrast, when we decrease the pressure smoothly, the density decreases initially, but remains constant when we decrease the pressure even further, as shown by the arrows in figure 4. This density jump and the corresponding hysteresis behaviour are indicative of a strong first-order vapour-liquid transition. The enormous density difference of the coexisting phases, as predicted by the mean-field theory, is probably responsible for the different results of the constant-pressure simulations upon compressing and diluting: as the maximum allowed volume change in the MC procedure is the volume itself, it is possible to compress the system in one step by many orders of magnitude while the reverse takes (too) many steps. Similar difficulties were encountered when we tried to simulate the two coexisting phases directly by using the Gibbs ensemble Monte Carlo method [8]. In this method the two coexisting phases are simulated simultaneously in two separate boxes, which can exchange particles and volume in order to obtain equal chemical potential and pressure in the two boxes. The constant-pressure simulations do not yield any hysteresis or a plateau value for $Z=20$, indicating that the homogeneous fluid phase is stable for all densities. For all values of $Z$ we observe, as expected, that for $\rho^{*} \rightarrow 0$ the pressure approaches the pressure of the ideal gas. More surprisingly, we also observe that at high density the pressure equals the ideal-gas pressure. Clearly, this is due to strong screening at high densities.

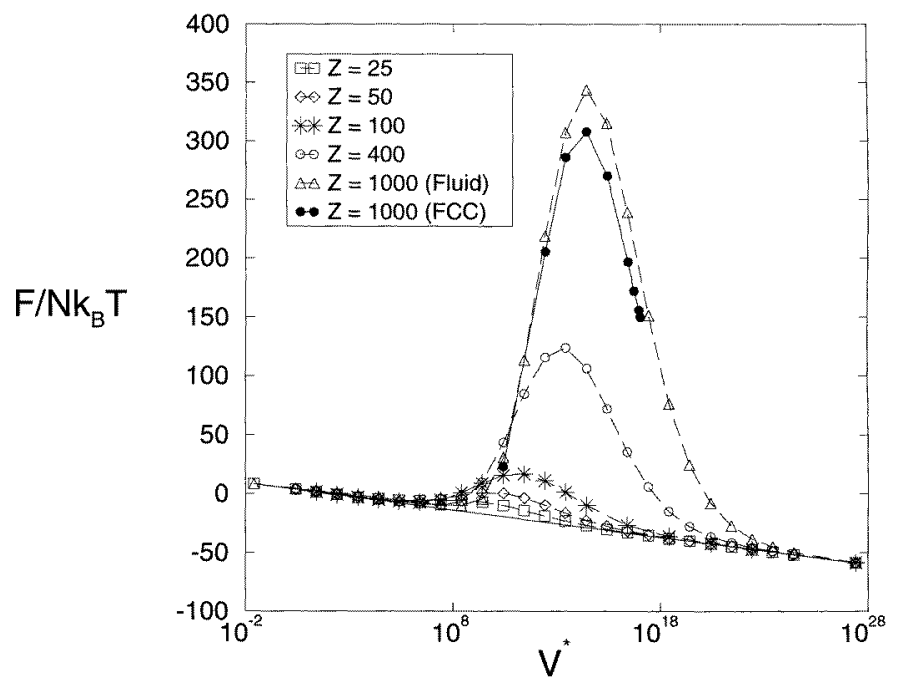

Figure 5. The fluid Helmholtz free energy per particle $F / N k_{B} T$ obtained by thermodynamic integration for $N=250$ as a function of the reduced volume $V^{*}=V / \lambda^{3}$ for $Z=$ $25,50,100,400$ and 1000 . For $Z=1000$ we also show the FCC Helmholtz free energy (-), which is clearly metastable. The straight line denotes the ideal-gas value.

In order to analyse the phase coexistence in more detail, we performed free-energy calculations using a thermodynamic integration technique [8]. The integration path starts at a non-interacting ideal gas at the desired density, proceeds by gradually switching on the Yukawa potential and finishes at the system of interest. We used 100 steps along the integration path and 1000 cycles per particle for each integration step. Each cycle consists 
of one displacement of a randomly selected particle. For several values of $Z$ we show the resulting Helmholtz free energy per particle, $F / N k_{B} T$, as a function of the reduced volume of the system $V^{*}=V / \lambda^{3}$ in figure 5 . We see that the free energy approaches the free energy of an ideal gas at low and at high densities, while it is non-ideal at intermediate densities. Fitting the free-energy curves enables us to compute the pressure and chemical potential as functions of reduced volume. By equating the pressures and the chemical potentials in the two coexisting phases, we determine the phase coexistence region. For $Z \leqslant 21.5$ we do not find a vapour-liquid coexistence, while for $Z \geqslant 22$ there is a two-phase region. The resulting vapour-liquid phase boundaries in the $\rho^{*}-Z^{-2}$ plane are indicated by the squares in figure 2.

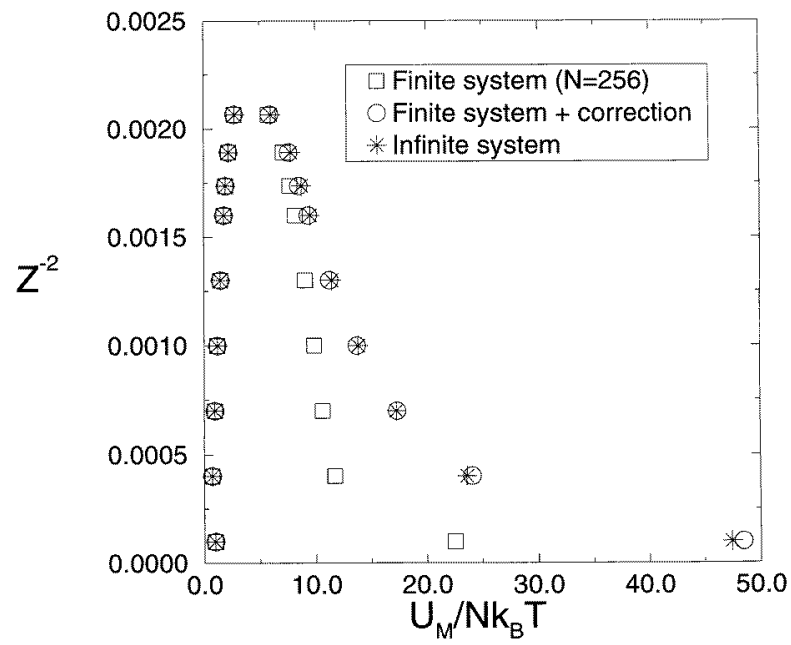

Figure 6. The Madelung energy $U_{M}$ for a finite system of $N=256$, uncorrected ( $\square$ ) and corrected $(O)$, and for an infinite system $(*)$, all three at the uncorrected phase boundaries.

A problem with the simulations presented so far is the fact that the finite number of $N=250$ particles is not sufficient to fully capture the long-range character of the interactions at low densities. It is not feasible to enlarge the number of particles to the extent that the theoretically predicted coexisting vapour phase of, say, $Z=50$ can be treated properly, as this would require $N>10^{6}$ for a $1 \%$ accuracy in the numerical determination of the potential energy. We have therefore corrected the free energies obtained from the thermodynamic integration by adding the mean-field expression (6) for the potential energy, with $r_{0}=V^{1 / 3} / 2$. This implies that $r_{0}$ is taken as half the length of the simulation box; the interactions within this distance have already been taken into account explicitly in the simulations. This correction should work well at low densities. The resulting corrected phase diagram is indicated by the crosses in figure 2 . We conclude immediately from comparing the uncorrected and the corrected simulation data that the correction affects the phase boundaries considerably, although hardly at the liquid side and only moderately near the critical point. The correction with $r_{0}=V^{1 / 3} / 2$ can be shown to give reliable results by comparing the Madelung energy $U_{M}$ of an infinite FCC lattice with that of a corrected and an uncorrected finite FCC lattice of $N=256$ particles. The Madelung energy, or the ground-state energy, is chosen because it can easily be enumerated exactly as a sum of the pair potentials over the FCC lattice with a lattice spacing dictated by the density. In figure 6 we show $U_{M}$ for the state points of the uncorrected vapour-liquid coexistence, where the 
low values of $U_{M}$ correspond to the well-screened liquid and the larger values to the longerranged vapour. Clearly, the uncorrected value of $U_{M}$ per particle in the finite system is identical to that of the infinite system in the liquid-state points, and even in the vapour near the critical point. Away from the critical point in the vapour, however, the uncorrected values deviate substantially from the exact ones. Applying the correction, based on (6) with $r_{0}=V^{1 / 3} / 2$, reduces the discrepancy almost to nothing, indicating that this mean-field correction is rather accurate. This is, moreover, a justification a posteriori for the use of this mean-field correction instead of a much more cumbersome alternative like the Ewald summation technique. We also wish to mention that a relatively small correction to the Helmholtz free energy, such as that applied near the critical point, has a profound effect on the precise location of the vapour-liquid equilibrium in the phase diagram.

We note, finally, that we did not find any evidence for crystalline FCC and BCC phases for $Z \leqslant 200$. These phases could, however, be formed within a density interval in the vapour-liquid coexistence region for $Z=400$ and $Z=1000$, but not outside this region. The free energy of a crystalline phase was determined by thermodynamic integration from an Einstein crystal to the actual system [8]. As an illustration, we display the (uncorrected) Helmholtz free energy of only the FCC phase for $Z=1000$ in figure 5. As expected from the mapping discussed above, the free energy of the FCC and BCC crystal is lower than that of the fluid at the same density. Nevertheless, the crystalline phase is unstable with respect to the vapour-liquid coexistence. Consequently, we have not found any evidence for a stable crystalline phase in the phase diagram.

\section{Discussion}

We have shown by using a simple mean-field theory and by computer simulations that a vapour-liquid transition is possible in a fluid with a purely repulsive Yukawa pair potential. This transition can therefore not be driven by the standard Van der Waals mechanism of liquid condensation, which is based on the existence of long-ranged attractions that provide cohesive energy. Instead, the mechanism here is provided by the dependence of the interparticle potential on the thermodynamic state of the system, as the screening parameter of the Yukawa fluid is density dependent. Consequently, the screening at high densities is so strong that ideal-gas behaviour is recovered. Thus the free energy at high densities is lowered to such an extent that it is favourable for a fluid at intermediate densities to phase separate into a very dilute gas and a dense liquid. Moreover, due to the good screening at high densities, no stable crystalline phase occurs in the phase diagram.

We wish to state explicitly that the currently discussed density-dependent screening mechanism is not the only one that may cause fluid-fluid phase separation in fluids of purely repulsive pair interactions. For instance, it has been argued that binary mixtures of small and large hard particles exhibit such phase separation due to the depletion mechanism [9]. Whether or not this mechanism is strong enough to actually induce phase coexistence in the binary hard-sphere mixture remains a matter of debate [10], but it is certainly strong enough in binary mixtures of small and large parallel cubes on a lattice, and in mixtures of thick and thin hard rods [11]. A second example of a mechanism for phase separation in a fluid with a purely repulsive pair interaction was recently given in reference [12], where the density dependence of the internal (free) energy of mutually repelling particles drives the phase separation into a dilute and a dense phase.

Finally, we mention that it is not obvious whether the density-dependent screening mechanism can in itself drive phase separation in experiments of salt-free colloidal suspensions. The reason for this is that the effective Hamiltonian of such a salt-free 
suspension cannot be written as a sum of effective density-dependent pair potentials, but also includes the density-dependent self-energy of the colloidal particles. This self-energy turns out to dominate the phase separation completely, as was shown in reference [12], while the density-dependent screening mechanism plays only a minor role. In order to show that this latter mechanism can also drive a phase separation, we considered here the rather academic case without the self-energy. There are also other reasons for which the model under consideration can be regarded as rather academic. For instance, we set the concentration of added salt to zero, whereas there is always a residual salt concentration of dissociated water molecules, which will affect the low-density behaviour of the fluid. Moreover, the high-density behaviour will be affected by the hard-core diameter of the colloidal particles, which we assume to be zero in the present case. In particular, a non-zero diameter will cause crystalline phases at sufficiently high densities for any $Z$. A non-zero diameter $D$ of the colloidal particles also tends to weaken the mechanism discussed in this paper, since the effective charge number increases by the DLVO factor $\exp [\kappa D / 2] /(1+\kappa D / 2)$ as the density increases (see (1)). Consequently, the repulsion at high densities will be relatively strong as compared to the point-particle case. Although the mechanism discussed in the present paper is therefore not the main one in these suspensions, it does contribute to the phase separation. In this light the present study must be seen as an illustration of a new mechanism that drives vapour-liquid phase separation in a fluid with purely repulsive potentials.

\section{Acknowledgments}

This work was made possible by financial support from the 'Région Rhône-Alpes' and from the EPSRC grant GR/K 80549.

\section{References}

[1] van der Waals J D 1873 On the continuity of the gaseous and liquid states PhD Thesis Hoogeschool te Leiden (Engl. Transl. Rowlinson J S 1988 Studies in Statistical Mechanics vol 14 (Amsterdam: North-Holland))

[2] Hagen M H J and Frenkel D 1994 J. Chem. Phys. 1014093

[3] Tejero C F, Daanoun A, Lekkerkerker H N W and Baus M 1994 Phys. Rev. Lett. 73752 Coussaert T and Baus M 1995 Phys. Rev. E 52862

[4] Verwey E J W and Overbeek J Th G 1948 Theory of the Stability of Lyotropic Colloids (Amsterdam: Elsevier)

[5] Robbins M O, Kremer K and Grest G S 1988 J. Chem. Phys. 883286

[6] Bitzer F, Palberg T, Löwen H, Simon R and Leiderer P 1994 Phys. Rev. E 502821

[7] Meijer E J and Frenkel D 1991 J. Chem. Phys. 942269

[8] Frenkel D and Smit B 1996 Understanding Molecular Simulations: From Algorithms to Applications (Cambridge: Academic)

[9] Asakura S and Oosawa F 1954 J. Chem. Phys. 221255 Vrij A 1976 Pure Appl. Chem. 48471

[10] Biben T and Hansen J-P 1991 Phys. Rev. Lett. 662215 Lekkerkerker H N W and Stroobants A 1993 Physica A 195387 Mao Y, Cates M E and Lekkerkerker H N W 1995 Phys. Rev. Lett. 754548 Götzelmann B, Evans R and Dietrich S 1998 Phys. Rev. E submitted

[11] Dijkstra M and Frenkel D 1994 Phys. Rev. Lett. 72298 Dijkstra M, Frenkel D and Hansen J-P 1994 J. Chem. Phys. 1013179 van Roij R and Mulder B M 1996 Phys. Rev. E 546430 Dijkstra M and van Roij R 1997 Phys. Rev. E 565594

[12] van Roij R and Hansen J-P 1997 Phys. Rev. Lett. 793082 